for the Special Issue of Biocatalysis in Spain

Submitted to Biocatalysis and Biotransformation

ID: GBAB-17-1067 Reviewed version

\title{
The making of versatile peroxidase by directed
}

\section{evolution}

David Gonzalez-Perez ${ }^{1} \&$ Miguel Alcalde ${ }^{*}$

${ }^{1}$ Department of Biocatalysis, Institute of Catalysis, CSIC, 28049 Madrid, Spain

* Corresponding author: malcalde@icp.csic.es

Running title: Directed VP evolution 


\section{ABSTRACT}

Versatile peroxidase (VP) secreted by white-rot fungi is involved in the degradation of lignin within land ecosystems. With a broad substrate scope and minor requirements, $\mathrm{VP}$ is an extremely attractive blueprint to be designed by the directed evolution tool-box. In recent years, improved VP variants have been generated that meet industrial requirements in terms of improved heterologous functional expression and activity, as well as stability at high temperatures or in the presence of strong inhibitors. This review describes the making of VP by directed evolution, addressing the most important findings and challenges that were faced, along with the future prospects for this research field.

Keywords: Versatile peroxidase, directed evolution, Saccharomyces cerevisiae, functional expression, stability, activity. 


\section{INTRODUCTION}

Lignin is a highly persistent biopolymer that gives consistency to the plant cell wall. Acting like a molecular mortar, lignin imbricates between cellulose and hemicellulose fibers providing protection against several external factors, including microbial attack (Martínez et al. 2005; Ruiz-Dueñas and Martinez 2009; Wong 2009). Given that lignin represents $1 / 3$ of the total carbon content of the biosphere, its degradation is a crucial aspect of the carbon cycle (Ragauskas et al. 2006, 2014). Among the organisms capable of modifying lignin in nature, white-rot basidiomycetes have possibly developed the most efficient oxidative system yet reported, a system based on the action of extracellular oxidoreductases (ligninases) that complete the combustion of lignin to $\mathrm{CO}_{2}$ and $\mathrm{H}_{2} \mathrm{O}$ (Martinez 2009; Sigoillot 2012). The ligninolytic armory is formed mainly by Class II peroxidases (generic peroxidases, -GPs-; lignin peroxidases, -LiPs-; manganese peroxidases, -MnPs-; versatile peroxidases, VPs); decolorizing peroxidases -DyP- and unspecific peroxygenases -UPO-; laccases; and $\mathrm{H}_{2} \mathrm{O}_{2}$-supplying enzymes. Together these enzymes interact in a complex cascade, assisted by small diffusible metabolites derived from fungal activity during wood decay (Alcalde 2015; Ruiz-Dueñas and Martínez 2009; Martinez et al. 2005).

The deciphering of the whole process of lignin mineralization identified VP (EC 1.11.1.16) as an important piece of the puzzle. As a result, it has now become possible to resolve fundamental questions on the natural evolution of lignin degrading fungi, the enzymes of which are thought to be linked to the end of coal deposition on earth during the Jurassic period (Ayuso-Fernandez et al. 2017; Floudas et al. 2012). VP was first discovered around 20 years ago in the edible mushroom Pleurotus enryngii, and a few years later in Bjerkandera species (Camarero et al. 1996, 1999; Mester and Field 1998; 
Ruiz-Dueñas et al. 1999, 2001). Fueled by catalytic concentrations of $\mathrm{H}_{2} \mathrm{O}_{2}$, VP can oxidize a wide variety of molecules, ranging from low- to high-redox potential substrates including phenolic, non-phenolic and diazo compounds (Ruiz-Dueñas et al. 2009a). The overall structure of VP involves 11 a-helices that are sustained by four disulfide bridges and two structural calcium atoms (Pérez-Boada et al. 2005) (Figure 1). In terms of catalytic sites, like GPs, VP has an access channel that is open to the solvent and where low-redox potential substrates are oxidized. In addition, VP has a superficial catalytic tryptophan that, in its active state $\left(\operatorname{Trp}^{*+}\right)$, oxidizes both low-redox and more significantly, high-redox potential substrates through a long-range electron transfer pathway to the heme, like LiP. Last but not least, in the sagittal plane of the protein structure there is a small heme access channel where $\mathrm{Mn}^{2+}$ is oxidized to $\mathrm{Mn}^{3+}$, the latter acting as a diffusible oxidizer as also occurs in $\mathrm{MnP}$ (Ruiz-Dueñas et al. 2007, 2009a) (Figure 2).

From a biotechnological viewpoint, VP represents a wonderful template for protein engineers. It is a relatively compact enzyme yet very promiscuous, with three catalytic sites for the oxidation of a multitude of compounds. Conversely, VP finds plenty of potential applications, such as in the design of biosensors and analytic kits, in paper and pulp bio-bleaching, bioremediation, textile industry, organic synthesis or biofuel production (Knop et al. 2015; Martínez et al. 2009; Mendonça-Maciel et al. 2010; Pizzul et al. 2009; Yadav and Yadav 2015). Despite this potential, VP must be subjected to further engineering in order to meet industrial standards and to become a truely robust industrial biocatalyst. In the last couple of decades, progress in rational design has helped unveil the different oxidation sites and the mechanisms by which VP acts (the reader is referred to other works and reviews that comprehensively address these aspects (Bao et al. 2014; Morales 
et al. 2012; Pérez-Boada et al. 2005; Pogni et al. 2005; Ruiz-Dueñas et al. 2007, 2008, 2009a, 2009b; Sáez-Jiménez 2015a, 2015b, 2015c, 2016). In the current article we wish to summarize the journey we have taken in the past decade to tackle crucial issues associated with VP engineering by directed evolution, including: i) achieveing adequate functional expression in heterologous hosts in which directed evolution can be perform; ii) thermostability; iii) inactivation in the presence of $\mathrm{H}_{2} \mathrm{O}_{2}$; and iv) activity and instability at neutral/alkaline pHs.

\section{The kick-off: Directed evolution for functional expression in Saccharomyces cerevisiae and for thermostability}

Although heterologous functional expression of VP was reported in Emericella nidulans, Aspergillus niger and Phanerochaete chrysosporium (Coconi-Linares et al. 2015; Eibes et al. 2009; Lu-Chau et al. 2004), these may not be the most suitable hosts for directed evolution enterprises. Indeed, evolution platforms assisted by Saccharomyces cerevisiae or Escherichia coli are the preferred choices due to their ease of manipulation and adaptability to different high-throughput contexts (Pourmir and Johannes 2012). Unfortunately, all attempts to express a functional VP in E. coli failed, the enzyme ending up in inclusion bodies that required in vitro refolding and/or the help of fusion partners to obtain the soluble protein (Bao et al. 2012; Pérez-Boada et al. 2002). By contrast, S. cerevisiae is usually a good departure point for library construction, functional expression (secretion) and the screening of eukaryotic genes/proteins in directed evolution strategies, and the case of VP is not an exception (Garcia-Ruiz et al. 2014; Gonzalez-Perez et al. 2012; Mate et al. 2016). This yeast can perform the complex posttranslational modifications required by ligninases, including glycosylation, the formation of disulfide bridges, or $\mathrm{N}$ - and $\mathrm{C}$-terminal processing, while it 
maintains an active secretory pathway. Moreover, a full set of episomal vectors are available to rapidly recover the target gene. In addition, large mutant libraries can be readily obtained due to the high transformation efficiency $\left(10^{6}-\right.$ $10^{8}$ transformants/ $\mu \mathrm{g}$ DNA). Yet undoubtedly, the most remarkable feature of S. cerevisiae for synthetic biology and metabolic engineering experiments stems from its high frequency of homologous DNA recombination, in conjunction with an accurate proof-reading machinery (Krivoruchko et al. 2011; Nevoigt 2008). As such, the DNA double-strand break repair mechanism used by $S$. cerevisiae does not involve restriction and ligation, and it is also error-free. By harnessing this system, simple library creation methods can be designed to shuffle different parental genes, to focus the molecular evolution on given segments (as predicted by computational algorithms), or to splice different blocks in order to construct chimaeras, to name just a few possibilities. The rule of thumb for all these methods is to engineer appropriate overlapping areas that flank each DNA fragment and foster the in vivo splicing between these stretches (Gonzalez-Perez et al. 2012). Thus, a gene can be fully reconstructed and cloned in vivo in the presence of the linearized plasmid in a single transformation step. In S. cerevisiae, in vivo homologous recombination is mediated by the Rad51 recombinase and ancillary factors. In practical terms, a length of $\sim 40 \mathrm{bp}$ for each overlapping area is recommended to achieve a good compromise between transformation efficiency and the number of crossover events (Alcalde 2010; Symington 2002).

As the starting block for directed evolution to achieve functional expression in $S$. cerevisiae, we centered our interest on the allelic variant $v p l 2$ from Pleurotus eryngii (Garcia-Ruiz et al. 2012). The failure to detect secretion of native VP in yeast microcultures (96-well plates) hampered the use of highredox potential substrates like reactive black five or veratryl alcohol in a high- 
throughput screening (HTS) assay of directed evolution. Instead, the colorimetric substrate ABTS (2,2'-Azino-bis(3-ethylbenzothiazoline-6-sulfonic acid) was used for the HTS so that activity against low redox potential compounds could be preserved at both the catalytic Trp and the heme access channel. The poor initial secretion was enhanced by switching the native signal peptide of VP with the a-factor prepro-leader from S. cerevisiae, which is commonly used for the heterologous expression of foreign proteins in yeast. Through this approach, the a-VP fusion protein was incorporated into the yeast secretory pathway, which facilitated: i) its processing during extrusion into the endoplasmic reticulum (pre-leader cleaving by a signal peptidase), ii) the action of the KEX2 and STE13 proteases in the Golgi compartment (cleaving the pro-leader), and iii) the final packaging of mature VP into vesicles for exocytosis. In addition, the production of VP in microplate format for HTS was optimized by adjusting cultivation parameters like shaking, aeration, heme source, $\mathrm{Ca}^{2+}$ supplement or ethanol concentration (the latter to enhance cytoplasmic membrane permeability). In this way, it was possible to enhance secretion to $\sim 0.4 \mathrm{mg} / \mathrm{L}$.

To avoid incorporating neutral or deleterious mutations during the evolution process, the mutagenic loads and the use of in vivo DNA shuffling were strictly controlled to favor the accumulation of, on average, one beneficial mutation per round of evolution. Thus, after four generations of random mutation and shuffling, the final secretion mutant (R4) contained four mutations in the protein scaffold. These four mutations were introduced in the first and second round of evolution, and they were further recombined to generate the mutated backbone for secretion E37K-V160A-T184M-Q202L (Figure 3). By this strategy secretion was improved 129-fold, yielding $22 \mathrm{mg} / \mathrm{L}$ of active, soluble and stable VP which can now be easily translated to Pichia 
pastoris for overproduction in bioreactor (unpublished material). HTS for thermostability was introduced from the fourth cycle of evolution onwards, whereby the selective pressure was increased from $60^{\circ} \mathrm{C}$ ( $4^{\text {th }}$ generation) to $90^{\circ} \mathrm{C}$ (6 $6^{\text {th }}$ generation) (Garcia-Ruiz et al. 2010, 2012). This approach allowed three further stabilizing mutations (H39R-D213A-G330R) to be introduced into the final mutant $(2-1 \mathrm{~B})$, with an overall enhancement of $8^{\circ} \mathrm{C}$ in kinetic thermostability. In the breakdown of the improvements in terms of specific activity and secretion of the $\mathrm{R} 4$ mutant, we found that while the specific activity increased 2.5 -fold, its expression was enhanced $\sim 52$-fold. However, the enhanced thermostability of the $2-1 \mathrm{~B}$ mutant was at the cost of reducing its activity, an expected natural trade-off between these two properties. After the biochemical characterization of both variants we detected some interesting side effects. Although oxidative stability was not targeted in the evolution experiment, we observed a $\sim 5$-fold improved oxidative stability of the $\mathrm{R} 4$ secretion mutant accompanied by a noticeable increment in the $K_{\mathrm{m}}$ for $\mathrm{H}_{2} \mathrm{O}_{2}$. Likewise, the thermostability mutant 2-1B displayed remarkable stability at alkaline $\mathrm{pH}$ (with a residual activity above $60 \%$ at $\mathrm{pH} 9$ after $120 \mathrm{~h}$ of incubation), which is rather unusual in fungal peroxidases. These results allowed us to commence new directed evolution enterprises, aimed at i) improving the oxidative stability of the $\mathrm{R} 4$ secretion mutant and ii) rising activity at alkaline $\mathrm{pH}$ in the thermostable mutant 2-1B (Figure 3).

\section{Directed evolution for oxidative stability}

Although the inactivation of heme containing peroxidases by $\mathrm{H}_{2} \mathrm{O}_{2}$ is a longstanding problem, it remains rather poorly understood and it still limits the use of peroxidases in many biotechnological sectors (Martínez 2007; Valderrama et al. 2002). At the ferric resting state, heme-peroxidases are first activated by a single molecule of $\mathrm{H}_{2} \mathrm{O}_{2}$ to yield an oxidized catalytic 
intermediate called compound I (oxoferryl IV porphyrin $\Pi$-cation radical), releasing one molecule of water as the only by-product. The enzyme then catalyzes two consecutive one-electron oxidations of two reducing substrates, regenerating the ground (reduced) state through a second catalytic intermediate called compound II (oxoferryl) and with the concomitant production of a second molecule of water (Figure 4).

It is well known that the main limiting step within this catalytic cycle is the low conversion rate from compound II to the ground state, which allows the former to accumulate and react with a new molecule of $\mathrm{H}_{2} \mathrm{O}_{2}$, either in the presence (with an excess of $\mathrm{H}_{2} \mathrm{O}_{2}$ ) or absence (at catalytic concentrations of $\mathrm{H}_{2} \mathrm{O}_{2}$ ) of the reducing substrate. In such scenarios compound II is diverted from the main catalytic cycle, producing the highly reactive intermediate compound III, a FeIII superoxide radical complex that irreversibly inactivates the enzyme irrespective of the route followed (e.g. via heme bleaching or through structural damage provoked by the interaction with the reactive oxygen species -ROS- generated). This inhibition is known as suicide inactivation, because paradoxically, $\mathrm{H}_{2} \mathrm{O}_{2}$ is both the co-substrate and an inhibitor of the enzyme (Valderrama et al. 2002, 2010). Suicide inactivation of VP is even more complex because of the presence of several catalytic sites and intermediates (Figure 4), which to the best of our knowledge, makes VP the most sensitive peroxidase to peroxides yet reported (Ruiz-Dueñas et al. 2009a).

Typically, the oxidative stability of peroxidases can be engineered by replacing highly oxidizable amino acids (e.g. Met, Cys, Tyr, His, Trp) by lowerredox potential counterparts (e.g. Ala, Val, Ile) (Kim et al. 2001; Ogola et al. 2010; Sáez-Jiménez et al. 2015a; Valderrama et al. 2002), and by means of directed evolution (Cherry et al. 1999; Miyazaki-Imamura et al. 2003; Morawsky et al. 2001). Given that suicide inactivation is mechanism-based, 
we combined structure-guided experiments with directed evolution to tailor oxidative stability in VP (Gonzalez-Perez et al. 2014a). Our R4 departure mutant showed a 4-fold enhancement in the $K_{\mathrm{m}}$ for $\mathrm{H}_{2} \mathrm{O}_{2}$, allowing it to work at high efficiency in saturating $\mathrm{H}_{2} \mathrm{O}_{2}$ conditions (at $2 \mathrm{mM}$ of $\mathrm{H}_{2} \mathrm{O}_{2} \mathrm{R} 4$ has an activity of $~ 57000$ ABTS-Units/L in culture supernatants) (Garcia-Ruiz et al. 2012). Rather than using a HTS based essentially on the incubation of mutant libraries at high concentrations of $\mathrm{H}_{2} \mathrm{O}_{2}$, we developed an assay in which the $\mathrm{H}_{2} \mathrm{O}_{2}$ :enzyme molar ratio was carefully controlled. Through this approach, the selection of mutants with distinct oxidative stabilities that depend on activity and/or secretion was circumvented, while we progressively increased the oxidative stress over the course of evolution (up to $0.6 \mathrm{mM}$ of $\mathrm{H}_{2} \mathrm{O}_{2}$ ).

A focused evolution protocol named MORPHING (Mutagenic Organized Recombination Process by Homologous In vivo Grouping) (Gonzalez-Perez et al $2014 b)$ was one of the library creation methods developed for this study. Through MORPHING, we targeted specific regions for random mutagenesis and recombination (predicted by computational guidance) while keeping the remaining protein structure protected from evolution. Thanks to the high frequency of homologous recombination in S. cerevisiae and with the help of the homologous overhangs flanking each MORPHING segment, functional mutant libraries were easily constructed and screened for the desired function. Indeed, MORPHING has become an ideal method for engineering not only VP in yeast but also, for other ligninases like: UPO, to evolve its native signal leader for secretion, to modify the peroxygenative:peroxidative activity ratio, or to improve yields in the synthesis of human drug metabolites; laccases, for the synthesis of heteropolymeric dyes, or to enhance thermostability and activity against high redox mediators; and aryl-alcohol oxidases to identify consensus/ancestor mutations or to oxidize secondary 
alcohols (Gonzalez-Perez et al. 2014b; Mate et al. 2017; Molina-Espeja et al. 2014; Vicente et al. 2016; Viña-Gonzalez et al. 2015, 2016 and unpublished results).

In engineering VP, we first applied MORPHING to three independent segments that were selected after careful structural alignment with other peroxidases with better oxidative stability. The regions targeted, from 26 to 69 amino acids in length, were mainly located in the heme cavity, including the proximal His domain (Leu149-Ala174), the distal His domain (Leu28-Gly57) and the Met environment (Ile199-Leu268). The improved mutants were found in both the distal/proximal His domains but not in the Met environment that contained three of the four oxidizable methionines (Met247, Met262 and Met265). This result was confirmed after subjecting Met265 and Met262 to combinatorial saturation mutagenesis (CSM), where the mutant library landscape showed $95 \%$ of the clones to be inactive. Recently, improved oxidative stability by mutating Met residues in VP was only achieved when all four Met residues were simultaneously removed, albeit at the cost of its activity, in accordance with our observations (Sáez-Jiménez et al. 2015a).

Given that the S. cerevisiae recombination machinery was inefficient in recombining nearby mutations (e.g. E40K, T45A), to keep the evolutionary process moving we performed in vitro recombination, in conjunction with new rounds of MORPHING, DNA shuffling, error-prone PCR and IvAM (in vivo assembly of mutant libraries). After four generations of directed evolution we entered a dense scenario in which multiple improved VP variants with a diverse trade-off between oxidative stability and activity were identified (i.e. variants with notably improved half-life for $\mathrm{H}_{2} \mathrm{O}_{2}$ but decreased activity, or variants with milder improvements in half-life but more sustained activity). To achieve a reasonable compromise between oxidative stability and activity, we 
developed another in vivo DNA recombination tool called DNA $\mathrm{puzzle}_{\text {(Gonzalez- }}$ Perez et al. 2014a). With this domain engineering method, we selected mutant variants from the $2^{\text {nd }}$ to $4^{\text {th }}$ round of evolution in order to amplify up to 9 evolved sequence blocks from three independent segments of VP. As such, the mutations in these sequences could be recombined in vivo and relocated in other mutational environments in a drive to find new epistatic interactions. Screening of the DNA puzzle mutant library flagged dozens of functional clones, from which two mutants were finally chosen on the basis of their half-life and activity. While the AT-AT variant (D22N-T45A-E83G-I103V-G107S-P141AF186L) resulted from the recombination of three sequence blocks, the SIth mutant (N11D-G35K-E40K-T45A-S86R-P141A-F186L-T323I) was the product of four evolved sequence blocks. In the presence of 3,000 equivalents of $\mathrm{H}_{2} \mathrm{O}_{2}$, the half-life of these mutants extended from $4 \mathrm{~min}$ to 18 (AT-AT) and $33 \mathrm{~min}$ (SIth) (Figure 3). Moreover, there was a $6^{\circ} \mathrm{C}$ improvement in the kinetic thermostability of SIth, whereas AT-AT maintained the same thermostability as the parental type. In terms of kinetics, the catalytic efficiency of AT-AT and SIth for ABTS was lower than the parental type, although these values were still higher than those of the native a-VP expressed in S. cerevisiae. Activity at the $\mathrm{Mn}^{2+}$ site was negatively affected, with slower kinetics for the AT-AT mutant and almost complete suppression in SIth, probably related to the enhanced thermostability of this mutant and the mutations introduced close this catalytic site. Finally, there were minor differences in the catalytic efficiency for the oxidation of high-redox potential substrates at the catalytic tryptophan, whereas the kinetics for $\mathrm{H}_{2} \mathrm{O}_{2}$ were mostly conserved.

The mutations identified were analyzed through a comprehensive structural alignment with other tolerant peroxidases in order to reveal key structural determinants. In general terms, the regions and mutations mapped 
were responsible for improvements in oxidative stability by making VP more compact/robust, while modifying the different catalytic sites.

\section{Directed evolution for activity at alkaline pH}

One important drawback for the application of VP -and by extension to all ligninolytic peroxidases- as an industrial biocatalyst in many processes (ranging from pulp bleaching to organic synthesis) is its lack of activity/stability at neutral/alkaline $\mathrm{pH}$. The structural integrity of VP is dependent on the correct coordination of two structural calcium atoms located above (fairly accessible at the distal domain) and below (buried deeply at the proximal domain) the plane of the heme (Pérez-Boada et al. 2005) (Figure 1A). Indeed, 7 and 8 oxygen atoms from surrounding amino acids and water molecules enter into the coordination spheres of such structural distal and proximal calcium ions, respectively (Figure 1B). It is well known that neutral/basic $\mathrm{pH}$ and/or high temperatures can modify $\mathrm{Ca}^{2+}$ coordination spheres, provoking their release and therefore the loss of activity. Indeed, after distal $\mathrm{Ca}^{2+}$ depletion, the catalytic distal His 47 approaches the iron atom of the heme, which in turn switches from a penta- to hexa-coordination state (bis-histidyl heme iron complex in low spin state), collapsing the heme cavity and inactivating the enzyme (George et al. 1999; Laberge et al. 2003; Lu-Chau et al. 2004; Youngs et al. 2000).

Notably, the 2-1B mutant obtained through directed evolution aimed to improve secretion and thermostability overcame such $\mathrm{Ca}^{2+}$ depletion under alkaline conditions (Garcia-Ruiz et al. 2012). Recently, this effect was attributed to the mutated E37K-H39R-G330R backbone that stabilized both distal and proximal $\mathrm{Ca}^{2+}$, allowing them to be correctly coordinated at alkaline $\mathrm{pH}$ and hence, preserving the pentacoordinated high-spin $\mathrm{Fe}^{3+}$ (Sáez-Jiménez 
et al. 2016). Although 2-1B was stable at alkaline conditions, there was hardly any activity at its three catalytic sites at basic $\mathrm{pH}$. Accordingly, further directed evolution to enhance the activity of this variant at neutral/alkaline $\mathrm{pH}$ was undertaken, while conserving activity at acid pH (Gonzalez-Perez et al. 2016), implying a selection criterion during HTS based on the ratio of activity at $\mathrm{pH} 4$ to that at neutral/basic $\mathrm{pH}$. Clones with $>80 \%$ of the parental activity at $\mathrm{pH} 4$ and improved activity at alkaline $\mathrm{pH}$ were initially selected for further analysis, and the process was further accelerated by increasing the selective pressure from $\mathrm{pH} 6$ to 8.5. After only three rounds of evolution with this strategy, we obtained the BB-8 mutant (harboring the mutations E140GP182S-Q229P) that was active over an enhanced $\mathrm{pH}$ range and that displayed strong hyperactivation after incubation at alkaline $\mathrm{pH}$ (with a $\sim 3$-fold increase in activity) (Figure 3). The active $\mathrm{pH}$ range for $\mathrm{BB}-8$ was expanded considerably for several substrates, including ABTS, sinapic acid and guaiacol. Consequently, BB-8 was active in the acid range ( $\mathrm{pH} 3-4)$ and remarkably, in the $\mathrm{pH}$ interval from 5 to 9 in which the activity of the parental VP was negligible.

The kinetic parameters measured for ABTS revealed enhanced catalytic efficiency at acid $\mathrm{pH}$ as result of increased affinity, which permitted BB-8 to remain active at basic pHs. This effect was mostly attributed to the E140G mutation that enabled the mutant to work with similar catalytic efficiency at pH 6 as the parental type at pH 3.5, due to the widening of the heme channel. Whilst the activity against $\mathrm{Mn}^{2+}$ was diminished due to the P182S mutation introduced close to this catalytic site, this mutation offered the first experimental insight into the role of the $\mathrm{Mn}^{2+}$ site for the direct (non-mediated) oxidation of ABTS at neutral/basic $\mathrm{pH}$. Site-directed mutagenesis experiments subsequently focused on blocking this catalytic site and molecular docking 
studies supported this finding (Gonzalez-Perez et al. 2016). Alternatively, we did not observe any activity at the catalytic $\operatorname{Trp} 164$ at basic $\mathrm{pH}$ due to the fact that the reduction potential of the Trp164 radical decreases as the $\mathrm{pH}$ increases, hindering the oxidation of high-redox potential substrates at neutral/basic $\mathrm{pH}$. Bearing in mind that the $\mathrm{p} K_{\mathrm{a}}$ of $\operatorname{Trp}^{*+}$ is $\sim 4.3$ (a value that varies slightly in function of the microenvironment), it is fully uncharged and possibly catalytically inactive at neutral or basic $\mathrm{pH}$. For these reasons, the long-range electron transfer pathway from $\operatorname{Trp} 164$ to the heme is permanently cancelled out at $\mathrm{pHs}>5$, thereby diverting the oxidative route for the oxidation of low-redox potential substrates to the other two catalytic sites at the time that the oxidation of high-redox potential compounds is supressed.

\section{Conclusions and outlook}

Almost 10 years ago we set out to perform directed evolution of VP using only $S$. cerevisiae, both as main expression host and as a vital tool-box for the creation of a DNA diversity. This long shot paid-off, since apart from creating a reliable platform to efficiently perform directed evolution, we showcased a broad portfolio of VP variants with improved traits. This not only paved the way for the directed evolution of VP, enhancing its functional expression and stabilization, but it also allowed us to understand how to harness recombination in $S$. cerevisiae in order to design methods to create libraries suitable for laboratory evolution. Such libraries have helped us identify hidden properties of VP that bring this enzyme one step closer to meeting industrial standards. Far from rejoicing, we consider these results represent merely the beginning and we believe important future progress in VP engineering will include the directed evolution of ancestral VP and the engineering of an artificial ligninolytic secretome. The former will be aided by recent data on the ancestral lineages of ligninolytic peroxidases, where several 
nodes from the late Carboniferous period were computationally reconstructed and expressed to situate VP as a potential generalist in the midst of the lignin degrading enzyme route (Ayuso-Fernandez et al. 2017). The use of ancestral VP and modern counterparts to recreate this evolutionary journey in vitro by means of laboratory evolution will help answer key questions on the natural evolution of VP, while providing a new collection of evolved variants with unexpected properties (Alcalde 2015, 2017). In terms of engineering the first artificial ligninolytic secretome, we recently co-expressed evolved versions of VP and laccase in S. cerevisiae, overcoming several metabolic barriers and bottlenecks associated with expression (Gonzalez-Perez and Alcalde 2014). In the drive to design a prototype of the white-rot yeast (WRY), we have just added new evolved versions of UPO and aryl-alcohol oxidases to the secretome pool, which can be used for the production of biofuels and value added products from lignocellulose. In the long term, the WRY could become an invaluable vehicle to understand the complex process of lignin mineralization in nature.

\section{ACKNOWDLEGEMENTS}

This work was supported by grants from the European Union [FP7KBBE-2013-7-613549-INDOX] the COST Action [CM1303 Systems Biocatalysis] and the Spanish Government [BIO2016-79106-R-Lignolution].

\section{REFERENCES}

Alcalde M. 2010. Mutagenesis protocols in Saccharomyces cerevisiae by in vivo overlap extension. In: Bramman J. Editor. In vitro Mutagenesis Protocols, 3rd ed. Methods in Molecular Biology 634. New Jersey: Springer-Humana Press. pp.3-15. 
Alcalde M. 2015. Engineering the ligninolytic enzyme consortium. Trends Biotechnol 33:155-162.

Alcalde M. 2017. When directed evolution met ancestral enzyme resurrection. Microb Biotechnol 10:22-24.

Ayuso-Fernandez I, Martínez AT, Ruiz-Dueñas FJ. 2017. Experimental recreation of the evolution of lignin degrading enzymes from the Jurassic to date. Biotechnol Biofuels. In press.

Bao X, Liu A, Lu X, Li JJ. 2012. Direct over-expression, characterization and $\mathrm{H}_{2} \mathrm{O}_{2}$ stability study of active Pleurotus eryngii versatile peroxidase in Escherichia coli. Biotechnol Lett 34:1537-1543.

Bao X, Huang X, Lu X, Li JJ. 2014. Improvement of hydrogen peroxide stability of Pleurotus eryngii versatile ligninolytic peroxidase by rational protein engineering. Enzyme Microb Technol 54:51-58.

Camarero S, Bockle B, Martínez MJ, Martínez AT. 1996. Manganese-mediated lignin degradation by Pleurotus pulmonarius. Appl Environ Microbiol 62:1070-1072.

Camarero S, Sarkar S, Ruiz-Dueñas FJ, Martínez MJ, Martínez AT. 1999. Description of a versatile peroxidase involved in the natural degradation of lignin that has both manganese peroxidase and lignin peroxidase substrate interaction sites. J Biol Chem 274:10324-10330.

Cherry JR, Lamsa MH, Schneider P, Vind J, Svendsen A, Jones A, Pedersen AH. 1999. Directed evolution of a fungal peroxidase. Nat Biotechnol $17: 379-384$.

Coconi-Linares N, Ortiz-Vázquez E, Fernández F, Loske AM, Gómez-Lim MA. 2015. Recombinant expression of four oxidoreductases in Phanerochaete chrysosporium improves degradation of phenolic and non-phenolic substrates. J Biotechnol 209:76-84. 
Eibes GM, Lu-Chau TA, Ruiz-Dueñas FJ, Feijoo G, Martínez MJ, Martínez AT, Lema, JM. 2009. Effect of culture temperature on the heterologous expression of Pleurotus eryngii versatile peroxidase in Aspergillus hosts. Bioproc Biosyst Eng 32:129-134.

Floudas D, Binder M, Riley R, Barry K, Blanchette RA, Henrissat B, Martínez AT, Otillar R, Spatafora JW, Yadav JS, et al. 2012. The Paleozoic origin of enzymatic lignin decomposition reconstructed from 31 fungal genomes. Science 336:1715-1719.

Garcia-Ruiz E, Mate D, Ballesteros A, Martínez AT, Alcalde M. 2010. Evolving thermostability in mutant libraries of ligninolytic oxidoreductases expressed in yeast. Microb Cell Fact 9:17.

Garcia-Ruiz E, Gonzalez-Perez D, Ruiz-Dueñas FJ, Martínez AT, Alcalde M. 2012. Directed evolution of a temperature, peroxide and alkaline $\mathrm{pH}$ tolerant versatile peroxidase. Biochem J 441:487-498.

Garcia-Ruiz E, Mate DM, Gonzalez-Perez D, Molina-Espeja P, Camarero S, Martínez AT, Ballesteros AO, Alcalde M. 2014. Directed evolution of ligninolytic oxidoreductases: from functional expression to stabilization and beyond. In: Sergio Riva, Wolf-Dieter Fessner, Editors. Cascade Biocatalysis. Wiley-VCH Verlag GmbH \& Co. KGaA. pp.1-22.

George SJ, Kvaratskhelia M, Dilworth MJ, Thorneley RN. 1999. Reversible alkaline inactivation of lignin peroxidase involves the release of both the distal and proximal site calcium ions and bishistidine co-ordination of the haem. Biochem J 344:237-244.

Gonzalez-Perez D, Garcia-Ruiz E, Alcalde M. 2012. Saccharomyces cerevisiae in directed evolution: An efficient tool to improve enzymes. Bioeng bugs 3:172-177. 
Gonzalez-Perez D, Garcia-Ruiz E, Ruiz-Dueñas FJ, Martínez AT, Alcalde M. 2014a. Structural determinants of oxidative stabilization in an evolved versatile peroxidase. ACS Catal 4:3891-3901.

Gonzalez-Perez D, Molina-Espeja P, Garcia-Ruiz E, Alcalde M. 2014b. Mutagenic organized recombination process by homologous in vivo grouping (MORPHING) for directed enzyme evolution. Plos one 9: e90919.

Gonzalez-Perez D, Alcalde M. 2014. Assembly of evolved ligninolytic genes in Saccharomyces cerevisiae. Bioengineered 5:254-263.

Gonzalez-Perez D, Mateljak I, Garcia-Ruiz E, Ruiz-Dueñas FJ, Martínez AT, Alcalde A. 2016. Alkaline versatile peroxidase by directed evolution. Catal Sci Technol 6:6625-6636.

Kim YH, Berry AH, Spencer DS, Stites WE. 2001. Comparing the effect on protein stability of methionine oxidation versus mutagenesis: steps toward engineering oxidative resistance in proteins. Protein Eng Des Sel $14: 343-347$.

Knop D, Yarden O, and Hadar Y. 2015. The ligninolytic peroxidases in the genus Pleurotus: divergence in activities, expression, and potential applications. Appl Microbiol Biotechnol 99:1025-1038.

Krivoruchko A, Siewers V, Nielsen J. 2011. Opportunities for yeast metabolic engineering: Lessons from synthetic biology. Biotechnol J 6:262-276.

Laberge M, Huang Q, Schweitzer-Stenner R, Fidy J. 2003. The endogenous calcium ions of horseradish peroxidase $\mathrm{c}$ are required to maintain the functional nonplanarity of the heme. Biophys J 84:2542-2552.

Lu-Chau TA, Ruiz-Dueñas FJ, Camarero S, Feijoo G, Martínez MJ, Lema JM, Martinez AT. 2004. Effect of $\mathrm{pH}$ on the stability of Pleurotus eryngii 
versatile peroxidase during heterologous production in Emericella nidulans. Bioproc Biosyst Eng 26:287-293.

Martínez AT, Speranza M, Ruiz-Dueñas FJ, Ferreira P, Camarero S, Guillen F, Martinez MJ, Gutierrez A, del Rio JC. 2005. Biodegradation of lignocellulosics: microbial, chemical, and enzymatic aspects of the fungal attack of lignin. Int Microbiol 8:195-204.

Martínez AT. 2007. High redox potential peroxidases. In: Polaina $\mathrm{J}$ and MacCabe, AP, Editors. Industrial Enzymes. Springer. pp.477-488.

Martínez AT, Ruiz-Dueñas FJ, Martínez MJ, del Rio JC, Gutierrez A. 2009. Enzymatic delignification of plant cell wall: from nature to mill. Curr Opin Biotechnol 20:348-357.

Mate DM, Gonzalez-Perez D, Mateljak I, Gomez de Santos P, Vicente AI, Alcalde M. 2016. The pocket manual of directed evolution: Tips and tricks. In: Brahmachari G, Demain AL, Adrio JL, Editors. Biotechnology of microbial enzymes: Production, biocatalysis and industrial applications. Amsterdam: Elsevier. pp.185-214.

Mate DM, Palomino MA, Molina-Espeja P, Martin-Diaz J, Alcalde M. 2017. Modification of the peroxygenative:peroxidative activity ratio in the unspecific peroxygenase from Agrocybe aegerita by structure-guided evolution. Protein Eng Des Sel 1-8.

Mendonça-Maciel MJ, Castro e Silva A, Telles Ribeiro HC. 2010. Industrial and biotechnological applications of ligninolytic enzymes of the basidiomycota: a review. Electron J Biotech 13:14-15.

Mester T, Field JA. 1998. Characterization of a novel manganese peroxidaselignin peroxidase hybrid isozyme produced by Bjerkandera species strain BOS55 in the absence of manganese. J Biol Chem 273:1541215417. 
Miyazaki-Imamura C, Oohira K, Kitagawa R, Nakano H, Yamane T, Takahashi H. 2003. Improvement of $\mathrm{H}_{2} \mathrm{O}_{2}$ stability of manganese peroxidase by combinatorial mutagenesis and high-throughput screening using in vitro expression with protein disulfide isomerase. Protein Eng Des Sel $16: 423-428$

Molina-Espeja P, Garcia-Ruiz E, Gonzalez-Perez D, Ullrich R, Hofrichter M, Alcalde M. 2014. Directed evolution of unspecific peroxygenase from Agrocybe aegerita. Appl Environ Microbiol 80:3496-3507.

Morales M, Mate MJ, Romero A, Martínez MJ, Martínez AT, Ruiz-Dueñas FJ. 2012. Two oxidation sites for low redox-potential substrates: A directed mutagenesis, kinetic and crystallographic study on Pleurotus eryngii versatile peroxidase. J Biol Chem 287:41053-41067.

Morawsky B, Quan S, Arnold FH. 2001. Functional expression and stabilization of horseradish peroxidase by directed evolution in Saccharomyces cerevisiae. Biotechnol Bioeng 76:99-107.

Nevoigt E. 2008. Progress in metabolic engineering of Saccharomyces cerevisiae. Microbiol Mol Biol Rev 72:379-412.

Ogola HJ, Hashimoto N, Miyabe S, Ashida H, Ishikawa T, Shibata H Sawa Y. 2010. Enhancement of hydrogen peroxide stability of a novel Anabaena sp. DyP-type peroxidase by site-directed mutagenesis of methionine residues. Appl Microbiol Biotechnol 87:1727-1736

Pérez-Boada M, Doyle WA, Ruiz-Dueñas FJ, Martínez MJ, Martínez AT, Smith AT. 2002. Expression of Pleurotus eryngii versatile peroxidase in Escherichia coli and optimisation of in vitro folding. Enzyme Microb Technol 30:518-524.

Pérez-Boada M, Ruiz-Dueñas FJ, Pogni R, Basosi R, Choinowski T, Martínez MJ, Piontek K, Martínez AT. 2005. Versatile peroxidase oxidation of 
high redox potential aromatic compounds: Site-directed mutagenesis, spectroscopic and crystallographic investigations of three long-range electron transfer pathways. J Mol Biol 354:385-402.

Pizzul L, Castillo M del P, Stenstrom J. 2009. Degradation of glyphosate and other pesticides by ligninolytic enzymes. Biodegradation 20:751-759.

Pogni R, Baratto MC, Giansanti S, Teutloff C, Verdin J, Valderrama B, Lendzian F, Lubitz W, Vazquez-Duhalt R, Basosi R. 2005. Tryptophanbased radical in the catalytic mechanism of versatile peroxidase from Bjerkandera adusta. Biochemistry 44:4267-4274.

Pourmir A, Johannes TW. 2012. Directed evolution: selection of the host organism. Comput Struct Biotechnol J 2:e201209012.

Ragauskas AJ, Williams CK, Davison BH, Britovsek G, Cairney J, Eckert CA, Frederick WJ, Hallett JP, Leak DJ, Liotta CL, et al. 2006. The path forward for biofuels and biomaterials. Science 311:484-489.

Ragauskas AJ, Beckham GT, Biddy MJ, Chandra R, Chen F, Davis MF, Davison BH, Dixon RA, Gilna P, Keller M, et al. 2014. Lignin valorization: improving lignin processing in the biorefinery. Science 344:1246843.

Ruiz-Dueñas FJ, Martínez MJ, Martínez AT. 1999. Molecular characterization of a novel peroxidase isolated from the ligninolytic fungus Pleurotus eryngii. Mol Microbiol 31:223-235.

Ruiz-Dueñas FJ, Camarero S, Pérez-Boada M, Martínez MJ, Martínez AT. 2001. A new versatile peroxidase from Pleurotus. Biochem Soc T 29: 116-122.

Ruiz-Dueñas FJ, Morales M, Pérez-Boada M, Choinowski T, Martínez MJ, Piontek K, Martínez AT. 2007. Manganese oxidation site in Pleurotus 
eryngii versatile peroxidase: A site-directed mutagenesis, kinetic and crystallographic study. Biochemistry 46:66-77.

Ruiz-Dueñas FJ, Morales M, Mate MJ, Romero A, Martinez MJ, Smith AT, Martinez AT. 2008. Site-directed mutagenesis of the catalytic tryptophan environment in Pleurotus eryngii versatile peroxidase. Biochemistry 47:1685-1695.

Ruiz-Dueñas, FJ, Morales M, García E, Miki Y, Martínez MJ, Martínez AT. 2009a. Substrate oxidation sites in versatile peroxidase and other basidiomycete peroxidases. J Exp Bot 60:441-452.

Ruiz-Dueñas FJ, Pogni R, Morales M, Giansanti S, Mate MJ, Romero A, Martínez MJ, Basosi R, Martínez AT. 2009b. Protein radicals in fungal versatile peroxidase: Catalytic tryptophan radical in both Compound I and Compound II and studies on W164Y, W164H and W164S variants. J Biol Chem 284:7986-7994.

Ruiz-Dueñas FJ, Martinez AT. 2009. Microbial degradation of lignin: how a bulky recalcitrant polymer is efficiently recycled in nature and how we can take advantage of this. Microb Biotechnol 2:164-177.

Sáez-Jiménez V, Acebes S, Guallar V, Martinez AT, Ruiz-Dueñas FJ. 2015a. Improving the oxidative stability of a high redox potential fungal peroxidase by rational design. Plos one 10:e0124750.

Sáez-Jiménez V, Baratto MC, Pogni R, Rencoret J, Gutierrez A, Santos JI, Martinez AT, Ruiz-Dueñas FJ. 2015b. Demonstration of lignin-toperoxidase direct electron transfer: a transient-state kinetics, directed mutagenesis, EPR and NMR study. J Biol Chem 290:23201-23213.

Sáez-Jiménez V, Fernández-Fueyo E, Medrano FJ, Romero A, Martinez AT, Ruiz-Dueñas FJ. 2015c. Improving the pH-stability of versatile 
peroxidase by comparative structural analysis with a naturally-stable manganese peroxidase. Plos one 10:e0140984.

Sáez-Jiménez V, Acebes S, García-Ruiz E, Romero A, Guallar V, Alcalde M, Medrano FJ, Martinez AT, Ruiz-Dueñas FJ. 2016. Unveiling the basis of alkaline stability of an evolved versatile peroxidase. Biochem $\mathrm{J}$ 473:1917-1928.

Sigoillot JC, Berrin JG, Bey M, Lesage-Meessen L, Levasseur A, Lomascolo A, Record E, Uzan-Boukhrins. 2012. Fungal strategies for lignin degradation. Adv Bot Res 61:263-308.

Symington LS. 2002. Role of RAD52 epistasis group genes in homologous recombination and double-strand break repair. Microbiol Mol Biol Rev 66:630-670

Valderrama B, Ayala M, Vázquez-Duhalt R. 2002. Suicide inactivation of peroxidases and the challenge of engineering more robust enzymes. Chem Biol 9:555-565.

Valderrama B. 2010. Deactivation of hemeperoxidases by hydrogen peroxide: Focus on compound III. In: Torres E, Ayala M, Editors. Biocatalysis based on heme peroxidases: peroxidases as potential industrial biocatalysts. Heidelberg: Springer. pp.291-314.

Vicente AI, Viña-Gonzalez J, Santos-Moriano P, Marquez-Alvarez C, Ballesteros O, Alcalde M. 2016. Evolved alkaline fungal laccase secreted by Saccharomyces cerevisiae as useful tool for the synthesis of C-N heteropolymeric dye. J Mol Catal B-Enzym 134:323-330.

Viña-Gonzalez J, Gonzalez-Perez D, Ferreira P, Martinez AT, Alcalde M. 2015. Focused directed evolution of aryl-alcohol oxidase in Saccharomyces cerevisiae by using chimeric signal peptides. Appl Environ Microbiol $81: 6451-6462$. 
Viña-Gonzalez J, Gonzalez-Perez D, Alcalde M. 2016. Directed evolution method in Saccharomyces cerevisiae: Mutant library creation and screening. J Vis Exp 110:e53761.

Wong DW. 2009. Structure and action mechanism of ligninolytic enzymes. Appl Biochem Biotechnol 157:174-209.

Yadav M, Yadav HS. 2015. Applications of ligninolytic enzymes to pollutants, wastewater, dyes, soil, coal, paper and polymers. Environ Chem Lett 13:309-318.

Youngs HL, Moënne-Loccoz P, Loehr TM, Gold MH. 2000. Formation of a bis(histidyl) heme iron complex in manganese peroxidase at high $\mathrm{pH}$ and restoration of the native enzyme structure by calcium. Biochemistry 39:9994-10000. 


\section{FIGURE LEGENDS}

FIGURE 1. General structure of VP and $\mathbf{C a}^{2+}$ attachment. (A) VP crystal structure (PDB ID: 3FJW) highlighted in blue cartoon mode, with the heme group shown as sticks in CPK colors and the disulfide bridges as yellow sticks. White letters indicate the name of the secondary VP structures. (B) Distal and proximal $\mathrm{Ca}^{2+}$ coordination spheres. Amino acid residues are indicated in $\mathrm{CPK}$ ball and stick mode. Water molecules and $\mathrm{Ca}^{2+}$ ions are highlighted as red and orange spheres, respectively.

FIGURE 2. VP oxidation sites. VP crystal structure shown in surface mode with the relative electrostatic potentials. Heme groups are highlighted in CPK sticks mode. The catalytic Trp164 and Leu165 are represented in yellow CPK sticks mode. LRTE, Long-range electron transfer pathway. Glu36, Glu40 and Asp175 at the $\mathrm{Mn}^{2+}$ binding site are shown as CPK sticks. The His47 and Lys43 residues are involved in the heterolytic cleavage of the $\mathrm{H}_{2} \mathrm{O}_{2}$ molecule while His 169 residue acts as fifth ligand of $\mathrm{Fe}^{3+}$.

\section{FIGURE 3. Different directed evolution campaigns followed for VP}

engineering. Stars show the new mutations while the squares reflect the accumulated mutations. Green and gray boxes indicate the a-prepro-leader. $T_{50}$, the temperature at which the enzyme losses $50 \%$ of its total activity after a 10 min incubation; $\mathrm{H}_{2} \mathrm{O}_{2} t_{1 / 2}$, half-life in the presence of 3,000 equivalents of $\mathrm{H}_{2} \mathrm{O}_{2}$ at $25^{\circ} \mathrm{C} \mathrm{pH} 6.0$.

FIGURE 4. Catalytic cycle of VP. VP in its ground state is activated by $\mathrm{H}_{2} \mathrm{O}_{2}$ giving rise to compound I (VP-IA), which can be diverted to compound VP-IB corresponding to the tryptophanyl cation radical at the surface of the enzyme. Compound I oxidizes a first reducing substrate switching to compound II (VPIIA) which can also be diverted to the corresponding compound VP-IIB. Finally, 
compound II in any of its catalytic forms returns to the ground state after a second oxidation of a new reducing substrate. VP-IA and VP-IIA catalyze the oxidation of low-redox potential substrates (mostly phenolics) at the heme channel as well as the oxidation of $\mathrm{Mn}^{2+}$ at the $\mathrm{Mn}^{2+}$ binding site. VP-IB and VP-IIB catalyze the oxidation of both low- and high-redox potential compounds (e.g. veratryl alcohol, VA) at the catalytic Trp at the surface of the protein through a long range electron transfer route to the heme. 